\title{
Transport phenomena in an electrochemical rotating cylinder reactor
}

\author{
F. Tomasoni ${ }^{1}$, J. F. Thomas ${ }^{1}$, D. Yildiz ${ }^{1}$, J. van Beeck ${ }^{1}$ \\ \& J. Deconinck ${ }^{2}$ \\ ${ }^{1}$ Von Karman Institute for Fluid Dynamics, Belgium \\ ${ }^{2}$ Department of Electrical Engineering, \\ Vrije Universiteit Brussel, Belgium
}

\begin{abstract}
Electrochemical processes are at the heart of a wide variety of both basic and advanced industrial activities. In many industrial electrochemical applications high flow rates are applied such that the electrochemical process is convectivetransport-dependent. In order to obtain the mass and charge transfer from the solution to the surface of the electrodes the flow field characterization is therefore essential.

In view of understanding in detail such processes, this paper presents the preliminary characterization of the complex flow field that takes place in an electrochemical rotating cylinder reactor (short aspect ratio $\Gamma=1.7$ and wide gap $\eta=0.16$ ). For this purpose a combined experimental, numerical and theoretical approach is followed. The experimental characterization is performed using time-resolved Particle Image Velocimetry (PIV), a non-intrusive and laserbased experimental technique. Special attention is paid to the image processing and possible optical problems. The investigation is performed for different flow conditions, from moderate (1700) to high (13600) Reynolds number.

The experimental results have been compared to the velocity profiles obtained using RANS and DNS simulations.

The theoretical part of this study is to provide new analytical models based on the solution of the momentum boundary layer equation and of the mass and charge transport equations. The analytical velocity profile agrees well with the experimental data.
\end{abstract}

Keywords: rotating cylinder electrode, PIV, RANS, DNS. 


\section{Introduction and motivation}

Electrochemical processes are governed by multi-component mass, heat and charge transport in laminar and turbulent flow, that are often multi-phase due to gas evolution at the electrode. Furthermore the mass transfer boundary layer is about one order of magnitude smaller than the hydrodynamic boundary layer and this, with other side effects (such as side reactions, surface contamination effects, gas evolution), yields to an increase of the difficulty of the problem.

Besides that, in many industrial electrochemical techniques the electrode moves with respect to the solution. These systems are called hydrodynamic electrochemical processes. In these processes generally hydrodynamical and electrochemical coupling is implied, because the electrochemical phenomenon is convective transport dependent. The flow field characterization is therefore essential in order to obtain the mass and charge transfer from the solution to the surface of the electrodes.

The objective of the present work is the preliminary characterization of the complex flow field that takes place in an electrochemical rotating cylinder reactor.

The flow characterization (bulk velocity, vorticity, statistics) is obtained using time-resolved Particle Image Velocimetry (PIV §2). A continuous laser is used as a light source and the acquisition of the images is realized via a high speed camera. The investigation is performed for different flow conditions, from moderate to high Reynolds number. Numerical simulations (RANS §3.1) were run before deciding the PIV parameters. The results provided by DNS simulation $\S 3.2$ have then been compared with the experiments, with the aim of validating the used DNS code, for which the capability of capturing an unsteady and complex solution represents a very important task. The aim of the theoretical section $\S 4$ is to provide new analytical models based on the solution of the momentum boundary layer equation, necessary for the future development of the equations for mass and charge transport. In section $\S 5$ the results of the flow field characterization are presented and discussed, while the profiles provided by the different approaches are compared. The paper is closed in section $\S 6$ by the conclusions and the future development of this work.

\section{Experiments}

The experiments have been performed on a cylinder reactor, which geometry can be described in terms of non dimensional quantity: the radii ratio is $\eta=r_{i} / r_{o} \approx$ 0.16 and the aspect ratio is $\Gamma=h / d \approx 1.7$, where $d=r_{o}-r_{i}$ is the gap size. The top, bottom and outer cylinder walls are in Plexiglas, in order to allow optical access.

The velocity measurements have been performed from two measurement planes: the horizontal plane (Figure 1(a)), located in the middle of the cylinder height, and the meridional plane (Figure 1(b)) which includes the axis of the cylinder.

The cylinder was filled with a solution of demineralized water at room temperature and seeding particles (Polyamide particles) previously mixed. 

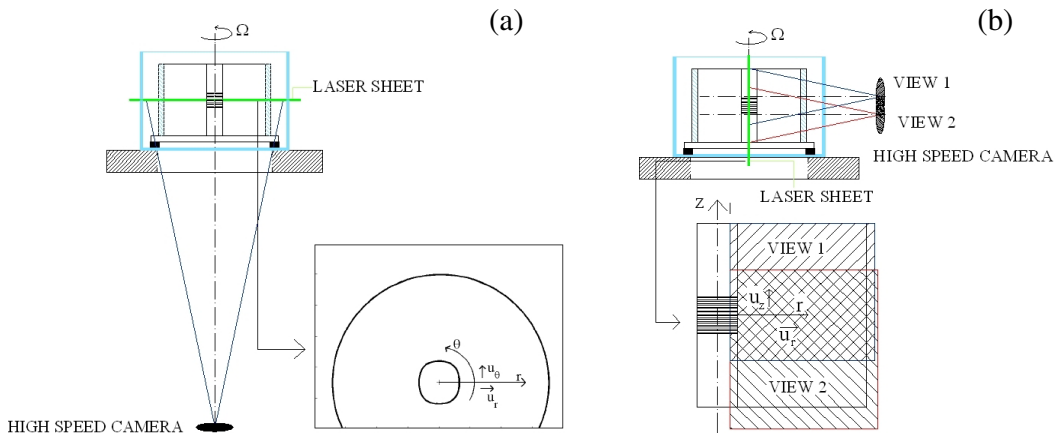

Figure 1: PIV: experimental setup. (a) horizontal plane measurement, and (b) meridional plane measurement.

The light source used in order to make visible the particles was a continuous laser (Coherent INNOVA 70C). By means of a spherical and a cylindrical lenses a narrow $(\approx 1 \mathrm{~mm})$ and intense ( $2 \mathrm{MW}$ single wavelength) light sheet has been obtained.

To acquire, display and record digital images, the Phantom v7.1 high speed camera has been used. The system is composed of: CMOS video sensor (800x600 pixels, 256 grey color) able to acquire images at a rate from 100 up to $160000 \mathrm{pps}$ and a PC equipped with a dedicated high speed camera software (Phantom 606). The maximum number of images acquirable consecutively was 1455 .

The inner cylinder was driven by a stepper motor (Maxon motor). The angular velocity of the inner cylinder has been varied from $\Omega_{i}=100 \mathrm{rpm}$ to $\Omega_{i}=800 \mathrm{rpm}$, in order to characterize the flow field that takes place in the cylinder reactor. The Reynolds number based on the gap $r_{o}-r_{i}, R e=\Omega_{i} r_{i}\left(r_{o}-r_{i}\right) / \nu$, varies from 1700 to 13600 .

\subsection{Measurement method: PIV}

Time-resolved Particle Image Velocimetry (PIV), a non-intrusive and laser-based experimental technique, has been used to experimentally characterized the flow field. Special attention is paid to the image processing and possible optical problems.

To compute and predict the separation time between the images, according to the basic PIV constraints, RANS simulations have been performed and from the resulting velocity field, the adequate separation time has been estimated.

Each acquisition contains 1454 velocity fields. A minimum non dimensional observation time $\bar{T}_{o}$ of 10 cylinder revolutions, where $\bar{T}_{o}=T_{o} \Omega_{i} /(2 \pi)$, is achieved in all the measurements. 


\section{Numerical simulation}

\subsection{RANS simulation}

The CFD software used to perform Reynolds Averaged Numerical Simulation is FLUENT, a flow modeling solver. The main objective is to have a reliable estimation of the velocity field, in order to use it as "predictor" for the experimental measurements. A second goal is represented by an a-posteriori validation of the Fluent results.

For this purpose steady-state, 2D, axisymmetric with swirl simulations have been performed. The grid used has $75 \times 100$ points in the radial and axial direction respectively. The rotational speed has been varied in the range of the studied velocities. In all the simulations the flow is considered turbulent and the $k-\varepsilon$ model is used.

\subsection{DNS simulation}

The numerical tool used in order to perform Direct Numerical Simulation is SFELES, a spectral/finite element code [1]. The simulation is performed on a 3D computational domain. A symmetric and a slightly asymmetric grids were used for the computation. The grids used have $100 \times 120 \times 64$ points in the radial, axial and azimuthal direction respectively.

The case analyzed is the one at $\mathrm{Re}=1700$. The unsteady simulation is realized with a time step $\Delta t=5 \cdot 10^{-3} \mathrm{~s}$, for a physical time of $T_{\mathrm{DNS}}=384 \mathrm{~s}$. The simulation required 55 hours running on 16 processors. The convergence is achieved approximately after 2000 iterations.

\section{Theoretical analysis}

\subsection{Flow modeling: boundary layer modeling}

The knowledge of the hydrodynamic boundary layer allows the study of the mass boundary layer and then, mass transfer at the electrode. Therefore the characterization of the studied boundary layer is essential for future developments. Thus, the aim of this section is to derive an expression for the mean tangential velocity profile in this layer.

Starting from the inner cylinder walls and moving in the radial direction till the center of the gap, the velocity profile is considered divided in three main regions: the wall region, the inertial sublayer and the core region. The subdivision is symmetric respect to the center of the gap.

The boundary layer equation is obtained starting from the continuity and the momentum equations in the azimuthal direction in cylindrical coordinates. Under the assumptions of axial symmetry and that the gradient of the axial stresses is 
small compared with the radial gradient of the azimuthal stresses, one obtains:

$$
r^{2}\left(\bar{\tau}_{r \theta}+\tau_{r \theta}^{t}\right)=\text { const }=r_{i}^{2} \tau_{w},
$$

where $\tau$ is the viscous shear stress tensor, $\tau^{\mathbf{t}}$ is the turbulent momentum flux tensor (the so-called Reynolds stresses) and $\tau_{w}$ is the wall shear stress.

\subsubsection{Near wall behavior}

In the vicinity of the wall the asymptotic behavior of the velocity profile has been often assumed linear with respect to the distance from the wall [2, 3].

In order to be coherent with what is observed in (1) and assuming that in the vicinity of the wall only the viscous terms play a role, it has to be stated:

$$
r^{2} \bar{\tau}_{r \theta}=r_{i}^{2} \tau_{w}
$$

Integrating the latter equation, with the adequate boundary condition, one gets:

$$
u_{\theta}=\Omega r-\frac{u_{\tau}^{2}}{2 \nu} \frac{r^{2}-r_{i}^{2}}{r}
$$

Observing equation (3), one can see that the tangential velocity close to the wall does not follow a purely linear trend. The linear behavior is therefore an approximation that is applicable only very close to the wall, that is when $r$ is very small, so that $\left(r+r_{i}\right) / r_{i} \approx 2$.

\subsubsection{Inertial sublayer}

In the inertial sublayer, often called logarithmic layer, the viscosity plays at most a minor role. Therefore, in this region, equation (1) reduces to:

$$
r^{2} \tau_{r \theta}^{t}=r_{i}^{2} \tau_{w}
$$

In order to obtain the time-smoothed velocity profile it is necessary to introduce a model for the turbulent momentum flux. In analogy with the Prandtl mixing length, a new model is here proposed:

$$
\tau_{r \theta}^{t}=-\rho k^{2}\left(r-r_{i}\right)^{2}\left|r \frac{d}{d r}\left(\bar{u}_{\theta} / r\right)\right| r \frac{d}{d r}\left(\bar{u}_{\theta} / r\right) .
$$

To close the equation, the continuity of the solution between two adjacent layer can be imposed. For example the expression of the average tangential velocity obtained imposing the continuity at $y^{+}=y_{s i}^{+}$, with the velocity obtained in (3), holds:

$$
\begin{aligned}
\bar{u}_{\theta}(r) & =\Omega r- \\
& -\frac{u_{\tau}}{k}\left[\frac{r}{r_{i}} \log \left[\left(\frac{r-r_{i}}{r}\right)\left(\frac{r_{s i}}{y_{s i}}\right)\right]+1-\frac{r}{r_{s i}}+\frac{u_{\tau} k}{2 \nu} \frac{r_{s i}^{2}-r_{i}^{2}}{r_{s i}^{2}} r\right],
\end{aligned}
$$

where $r_{s i}=y_{s i}^{+} \nu / u_{\tau}+r_{i}$ and $y_{s i}=r_{s i}-r_{i}=y_{s i}^{+} \nu / u_{\tau}$. 


\section{Results and discussion}

The tangential velocity profile represents the primary component of the motion in the RCE. Nevertheless, the topology of the flow is more complex. As it can be observed from Figure 2, the motion is not purely circular (the streamlines are not close in circles) as a radial velocity component exists.

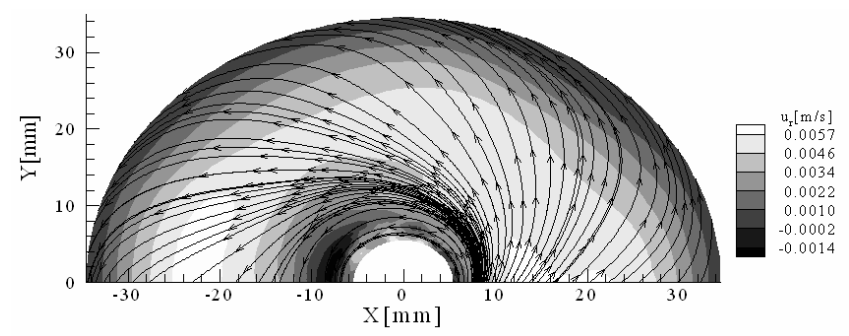

Figure 2: PIV (horizontal plane): $\operatorname{Re}=1700$. Average radial velocity contour.

It is possible to relate this behavior to the existence of an outgoing jet from the inner cylinder boundary layer, as it can be seen in Figure 7(a). For all the Reynolds numbers analyzed, in the average vector field, two big counter rotating cells are recognizable in the lateral plane.

A deeper understanding of the flow behavior can be obtained by analyzing the instantaneous velocity fields. In Figure 3(a), where an instantaneous field is reported for $\mathrm{Re}=1700$, one can observe two rollers. Observing the time evolution of the two cells, it appears that three different configurations are possible: a symmetric position of the rollers with respect to the middle height of the cylinder and an asymmetric one (one bigger than the other in both the direction). The latter configuration appears more frequently in the observed time history, so that the average velocity field keeps memory of the periodicity of the flow.

The situation is different for $\mathrm{Re}=13600$, Figure 3(b), where small turbulent structures are distributed in all the field and they coexist with bigger cells. The two coherent structures that appear in the average field find therefore their origin into an average-operation.

The average specific angular momentum $\mathcal{L}=u_{\theta} r / \Omega_{i} r_{i}^{2}$ (Figure 4) is constant in the core of the gap. As explained by [4], this means that, far from the wall, the flow is well mixed. As the Reynolds number increases the $\mathcal{L}$ value increases, till the value $\mathcal{L} \approx 0.45$, which has been found by [3] and [5], for fully turbulent flow conditions.

The normalized RMS (Figure 5) exhibits a peak close to the inner cylinder wall, where it exhibits always the maximum value near $10 \%$. A maximum normalized RMS of $7 \%$ characterizes the central region of the gap. In all the cases the velocity fluctuation profiles are maximum at the middle height of the reactor that is the interface region between the two rollers. 


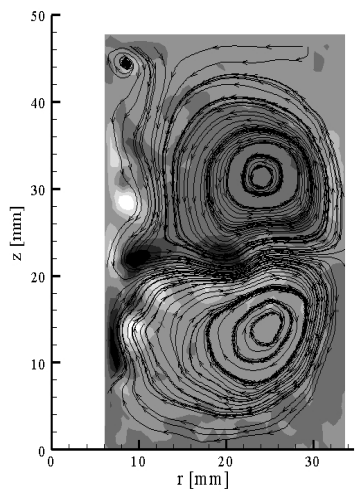

(a)

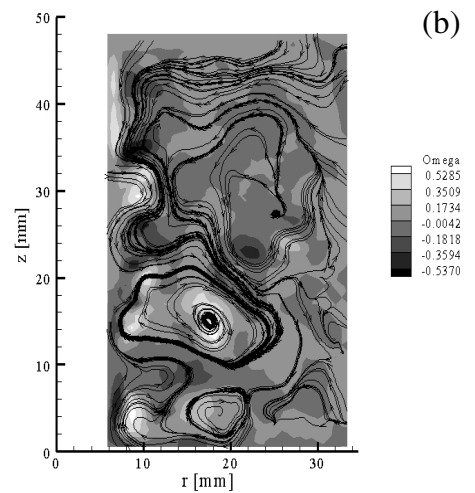

Figure 3: PIV: vorticity, instantaneous field: (a) $\operatorname{Re}=1700$, and (b) $\operatorname{Re}=13600$.

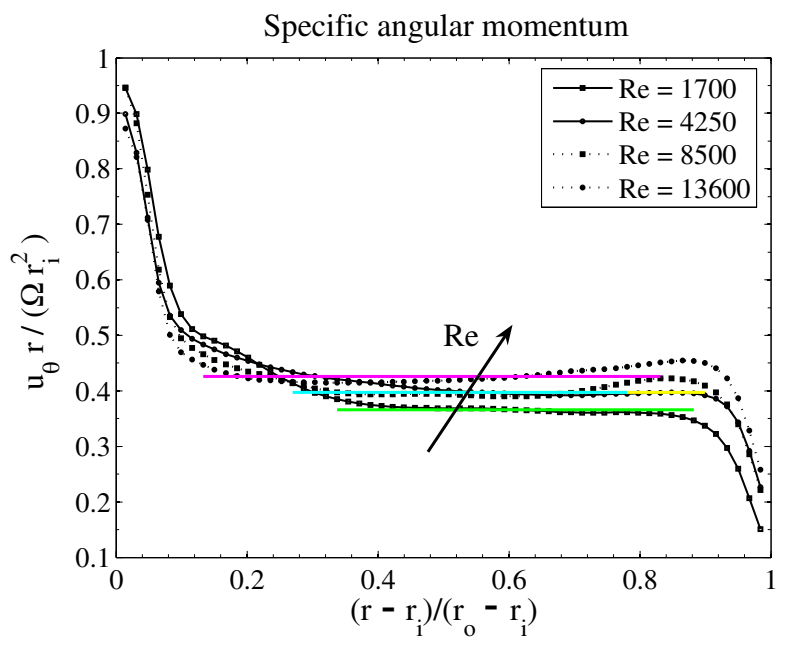

Figure 4: Average specific angular momentum $\mathcal{L}=u_{\theta} r / \Omega_{i} r_{i}^{2}$.

\subsection{Comparison: PIV and analytical models}

An iterative method is employed in order to compare the analytical velocity profiles with the experimental data. The validation of the viscous sublayer velocity profile is particularly difficult, because the latter region extends in a very small region close to the wall (less than $1 \mathrm{~mm}$ ) where the PIV measurements risk to be compromised.

As it is possible to observe in Figure 6, the analytical velocity profile agrees well with the experimental data. 


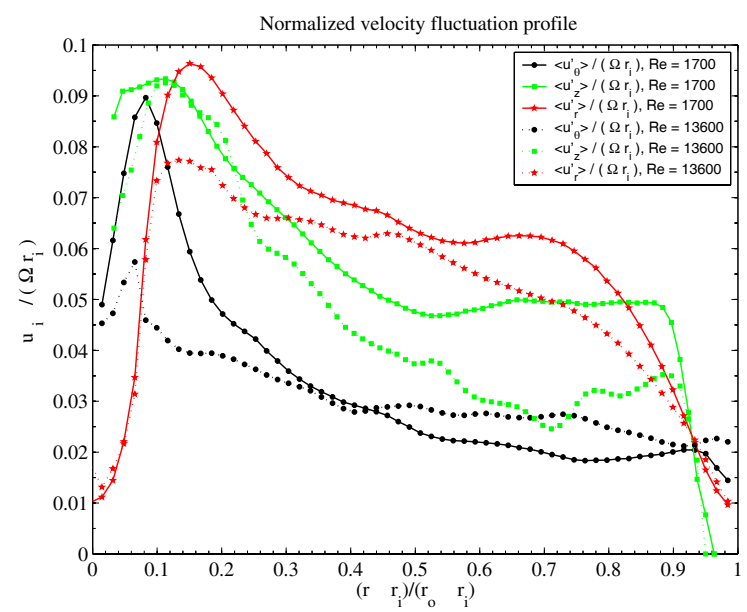

Figure 5: Normalized RMS.

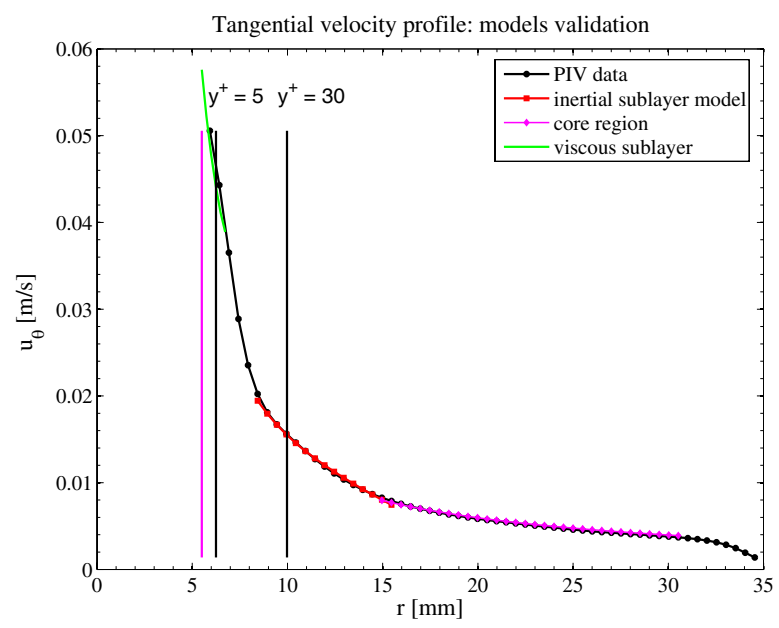

Figure 6: Average tangential velocity profile: models preliminary validation with PIV data.

\subsection{Comparison: PIV and numerical simulations}

The numerical simulations are compared both in a qualitative (topology) and quantitative way to the experimental results, for $\mathrm{Re}=1700$. From Figure 7 , it is possible to see that in the average field, all the data show two counter rotating cells. While in the experiments and in the DNS simulation with a slightly asymmetric grid, the two rollers are not symmetric with respect to the middle of 
the cylinder height, in the case of the RANS simulation and the DNS simulation with a symmetric grid, the coherent structures are symmetric. The asymmetry of the two rollers can be explained again by looking to the instantaneous behavior of the flow. For $\mathrm{Re}=1700$, the flow is not fully turbulent and an unstable regime seems to be established in the cell. A small perturbation is sufficient to trigger the asymmetry of the two rollers. In the DNS simulation, the asymmetry of the grid induces such a "perturbation". From the quantitative comparison of the velocity profiles, it appears that the maximum difference between the experimental and the numerical data is of the order of $20 \%$.

(a)
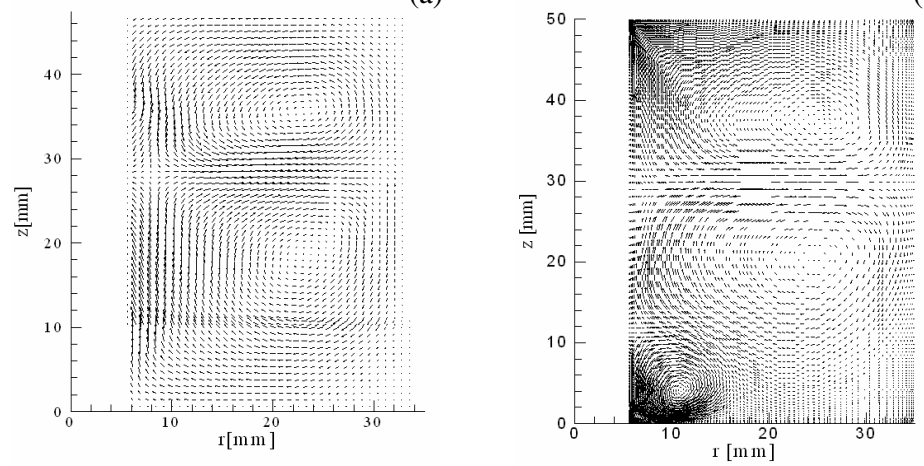

(b)

(c)

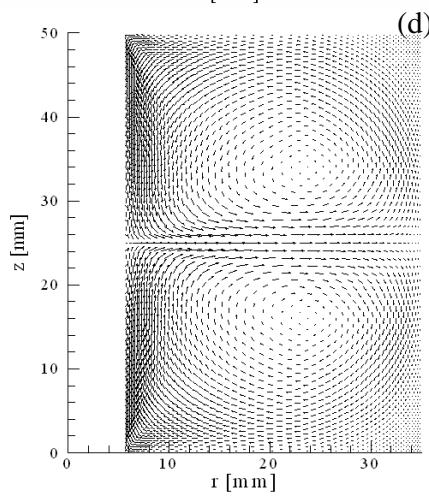

Figure 7: Average vector field, (a) Re $=1700$. PIV, (b) DNS not symmetric grid, (c) DNS symmetric grid, (d) RANS. One vector out of two is plotted.

\section{Conclusions and future work}

The average flow field shows, in the range of the Reynolds studied, two big counter rotating structures in the meridional plane. Those cells are characterized by radial inflow at the cylinder endwalls, while an outgoing jet constitutes the interface line 
between the rollers themselves. Furthermore, a core region of constant angular momentum (the specific angular momentum found is $0.37-0.45$ ) is observed. This indicates that a region of well mixed flow exists in the center of the gap.

In both the flow condition nevertheless, the normalized RMS never exceeds $11 \%$ as peak value and $7 \%$ in the core region.

The results of the numerical simulations are in qualitative agreement with the measurement results, but an influence on the grid can be observed. For $\mathrm{Re}=1700$, a symmetric grid provides two symmetric rotating structures, while a slightly asymmetric grid gives two non symmetric rollers. The grid asymmetry acts therefore as a perturbation that triggers the asymmetry of the two structures.

The preliminary validation of the analytical models for the average tangential profile in the boundary layer gives satisfactory results. Having completed the validation of the models of the velocity, it will be possible to develop the model for the concentration profile in the mass boundary layer.

\section{Acknowledgement}

We acknowledge the support from the Instituut voor de aanmoediging van innovatie door Wetenschap \& Technologie in Vlaanderen (IWT, contract nr. SBO 040092).

\section{References}

[1] Detant, Y. et al, Sfeles manual. Technical report, VKI, VUB, BYU, 2006.

[2] Bilson, M. \& K., B., Comparison of turbulent scalar transport in a pipe and a rotating cylinder. Third International Conference on CFD in the Minerals and Process Industries, pp. 493-498, 2003.

[3] Lathrop, D., Fineberg, J. \& Swinney, H., Transition to shear-driven turbulence in couette-taylor flow. Physical Review A, 46, pp. 6390-6408, 1992.

[4] King, G., Li, Y., Lee, W., Swinney, H. \& Marcus, P., Wave speeds in wavy taylor-vortex flow. J Fluid Mech, 141, pp. 365-390, 1984.

[5] Lewis, G. \& Swinney, H., Velocity structure functions, scaling, and transitions in high-reynolds-number couette-taylor flow. Physical Review E, 59, pp. 5457-5467, 1999. 